\title{
A Novel Particle Swarm Optimization for Optimal Scheduling of Hydrothermal System
}

\author{
Wenping Chang ${ }^{1,2}$ \\ ${ }^{1}$ School of Electrical Engineering, Xi'an Jiaotong University, Xi'an, China \\ ${ }^{2}$ Henan Mechanical and Electrical Engineering College, Xinxiang, China \\ E-mail: wpchang@163.com \\ Received April 18, 2010; revised July 5, 2010; accepted August 14, 2010
}

\begin{abstract}
A fuzzy adaptive particle swarm optimization (FAPSO) is presented to determine the optimal operation of hydrothermal power system. In order to solve the shortcoming premature and easily local optimum of the standard particle swarm optimization (PSO), the fuzzy adaptive criterion is applied for inertia weight based on the evolution speed factor and square deviation of fitness for the swarm, in each iteration process, the inertia weight is dynamically changed using the fuzzy rules to adapt to nonlinear optimization process. The performance of FAPSO is demonstrated on hydrothermal system comprising 1 thermal unit and 4 hydro plants, the comparison is drawn in PSO, FAPSO and genetic algorithms (GA) in terms of the solution quality and computational efficiency. The experiment showed that the proposed approach has higher quality solutions and strong ability in global search.
\end{abstract}

Keywords: Hydrothermal Generation Scheduling, Particle Swarm Optimization, Fuzzy, Adaptability

\section{Introduction}

Optimal operation is one of the important optimization issues in a hydrothermal power system. Its objective is to minimize the operation cost of thermal units in a given period of time while all constraints are satisfied. Since the cost of power generation is huge, the economic consequence of operation scheduling is significant. A number of methods have been proposed for solving the hydrothermal scheduling problem. Some examples of these methods are nonlinear programming (NLP) [1-3], Dynamic Programming (DP) [4-6], Lagrangian Relaxation (LR) [7-9], Tabu Search [10], Expert Systems [11], Artificial Neural Networks (ANN) [12], Genetic Algorithms (GA) [13-15]. Among these methods, DP would provide a good framework for optimizing the decisions. The main difficulty with DP is the curse of dimensionality that is the exponential increase in computation, it suffers from the "curse of dimensionality". The LR has shown great potential for the optimal problem, but the disadvantage of LR is its inherent sub-optimality.

Particle swarm optimization (PSO) is an evolutionary computation technique [16], it has lots of advantage: simple concept, easy implementation, robustness to control parameters and computational efficiency. Recently,
PSO have been successfully used in many areas [17-21]. Although PSO has many strong points, it has some shortcoming such as premature convergence. To overcome these problems, many methods have been developed. Shi and Eberhart introduced an inertia weight in 1998 [22], the inertia weight method could improve the performance of algorithm, but a fixed inertia weight does not satisfy the balance between global and local search, so many attempts have been tried by using various inertia weight strategies to make it performance better. Shi and Eberhart proposed a linear decreasing strategy in 1999 [23], which does not truly reflect the actual search process to find the optimum.

In this paper, a fuzzy adaptive particle swarm optimization (FAPSO) is proposed to the operation of hydrothermal power system, which is designed to dynamically adjust the inertia weight as the environment changing. This can improve the global and local search ability of the PSO and overcome the disadvantages of the PSO. The correlative examination indicates that the FAPSO has fast convergent velocity and powerful search capabilities to generate satisfactory results.

\section{Problem Formulation}

The objective of optimal operation to hydrothermal power 
system is usually to minimum the thermal cost function, while satisfying physical and operational constraints.

\subsection{Objective Function}

Hydrothermal scheduling is the optimization of a problem with non-linear objective function, the objective function to be minimized can be written as:

$$
\begin{aligned}
\min J & =\sum_{t=1}^{T} \sum_{i=1}^{N} F_{i}\left(P_{t h}(i, t)\right) \\
& =\sum_{t=1}^{T} \sum_{i=1}^{N}\left[a_{i} P_{t h}^{2}(i, t)+b_{i} P_{t h}(i, t)+c_{i}\right]
\end{aligned}
$$

where $T$ is number of operating periods, $N$ is number of thermal plants, $F$ is composite cost function, $P_{t h}(i, t)$ is loading of $i$ th thermal unit at time $t, a_{i}, b_{i}, c_{i}$ are thermal generation cost coefficients.

\subsection{Constraints}

The constraints must be satisfied the optimization process, which are as follows:

1) Total generation meets the system demand

$$
\sum_{i \in N} P_{t h}(i, t)+\sum_{j \in M} P_{h}(j, t)=P_{D}(t)
$$

where $M$ is number of hydropower stations, $P_{h}(j, t)$ is loading of $j$ th hydro unit at time $t, P_{D}(t)$ is load demand at time $t$.

2) System spinning reserve limits

$$
\sum_{i \in N} R_{s}(i, t)+\sum_{j \in M} R_{h}(j, t)-P_{D}(t) \geq R_{r e q}(t)
$$

where $R_{r e q}(t)$ is required system spinning capacity reserve.

3) Thermal plant loading limits

$$
P_{i, \text { min }} \leq P_{t h}(i, t) \leq P_{i, \text { max }}
$$

where $P_{i, \min }$ is minimum power output of thermal unit $i$, $P_{i, \max }$ is maximum power output of thermal unit $i$.

4) Ramp rate constraints

$$
P_{t h}(i, t+1)-\Delta_{i} \leq P_{t h}(i, t) \leq P_{t h}(i, t+1)+\Delta_{i}
$$

where $\Delta_{i}$ is ramp rate maximum permitted for the thermal unit $i$.

5) Hydro plant loading limits

$$
P_{j, \min } \leq P_{h}(j, t) \leq P_{j, \max }
$$

where $P_{j, \min }$ is minimum power output of hydro unit $j$, $P_{j, \max }$ is maximum power output of hydro unit $j$.

6) Reservoir level limits

$$
V_{j, \min } \leq V(j, t) \leq V_{j, \max }
$$

where $V_{h}(j, t)$ is storages of reservoir $j$ during time $t$, $V_{j, \min }$ is minimum storage of reservoir $j, V_{j, \max }$ is maximum storage of reservoir $j$.

7) Reservoir discharge limits

$$
Q_{j, \text { min }} \leq Q(j, t) \leq Q_{j, \max }
$$

where $Q(j, t)$ is water discharge of reservoir $j$ at time $t$, $Q_{j, \min }$ is minimum release of reservoir $j, Q_{j, \max }$ is maximum release of reservoir $j$.

8) Water balance equation

$$
V(j, t+1)=V(j, t)+I(j, t)+\sum_{j \in \Omega_{i}} Q\left(j, t-\tau_{j l}\right)-Q(j, t)
$$

where $I(j, t)$ is inflow of reservoir $\mathrm{j}$ during time $t, \tau_{j l}$ is water delay time between reservoir $j$ and $i$.

9) Initial and terminal reservoir levels

$$
\begin{aligned}
& V(0)=V^{0} \\
& V(T)=V^{T}
\end{aligned}
$$

where $V_{j}^{0}$ is initial storages of reservoir $j, V_{j}^{T}$ is terminal storages of reservoir $j$.

10) Power generation of hydro plant

The hydro generator power output is written in terms of reservoir volume instead of the reservoir net head and the rate of water discharge, $Q$, a frequently used functional is

$$
\begin{aligned}
& P_{h}(j, t)=c_{j, 1} V^{2}(j, t)+c_{j 2} Q^{2}(j, t)+c_{j 3} V(j, t) Q(j, t) \\
& +c_{j 4} V(j, t)+c_{j 5} Q(j, t)+c_{j 6}
\end{aligned}
$$

where $C_{j}^{1}, \cdots, C_{j}^{6}$ are hydro power generation coefficients.

\section{Implementation of Fapso for Optimization Problem}

\subsection{Overview of the PSO}

PSO is a population-based optimization algorithm, which was firstly proposed by Kennedy and Eberhant in 1995 [24]. It simulates the food searching activities of a swarm of birds (particles), and every particle has its own location and velocity, individuals are evolved by cooperation and competition among themselves through generations, particles move around the search space until they find the optimal solution.

In n-dimensional search space, the location of the particle is represented as the vector $X_{m}=\left(x_{m 1}, x_{m 2}, \cdots, x_{m n}\right)$, and its velocity is represented as the vector $V_{m}=\left(v_{m 1}, v_{m 2}, \cdots, v_{m n}\right) . P_{\text {best }}$ and $G_{\text {best }}$, respectively, are the best location of individual $m$ and all particles' best location so far. Using the information, the velocity of 
individual is modified as shown below:

$$
\begin{aligned}
& V_{m}^{k+1}=\omega^{k+1} V_{m}^{k}+a_{1} \operatorname{rand}() \times\left(P_{m b e s t}^{k}-X_{m}^{k}\right) \\
& +a_{2} \operatorname{rand}() \times\left(G_{m b e s t}^{k}-X_{m}^{k}\right)
\end{aligned}
$$

where $a_{1}, a_{2}$ are learning factors.

Using (12), a certain velocity that gradually gets close to $P_{\text {best }}$ and $G_{\text {best }}$ can be calculated. Each individual moves from the current location to the next one using the following equation:

$$
X_{m}^{k+1}=X_{m}^{k}+V_{m}^{k+1}
$$

The search mechanism of the PSO using the modified velocity and location of individual $m$ is showed in Figure 1.

In PSO, it is the inertia weight to balance between global and local search ability. A large inertia weight facilitates a global search while a small inertia weight facilitates a local search, concept of linearly decreasing inertial weight for particle swarm optimization (LDWPSO) is introduced in [23], which $\omega$ is given by

$$
\omega=\omega_{\max }-\frac{i \operatorname{ter}\left(\omega_{\max }-\omega_{\min }\right)}{\text { iter }_{\max }}
$$

where $\omega$ is inertia weight, iter is the number of iterations, iter $_{\text {max }}$ is the maximum number of iterations.

In the LDWPSO approach, particle's fitness is best until the preceding iteration, its velocity is kept unchanged in the next iteration, otherwise, the particle's velocity and position are changed according to (12) and (13), which does not truly reflect the search process to find the optimum.

In order to obtain a better search process, the inertia weight should be nonlinearly, dynamically changed to balance between global and local search ability. Therefore, fuzzy adaptive PSO is presented to design a fuzzy system to dynamically adjust the inertia weight.

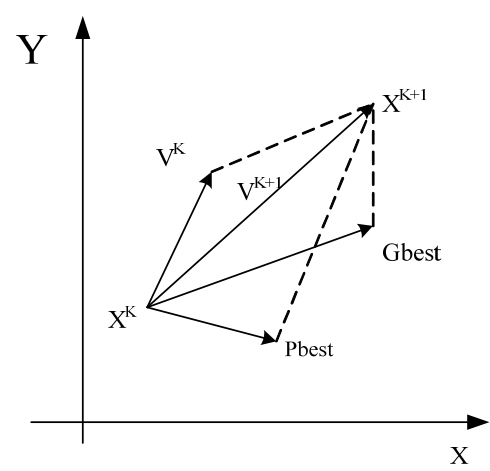

Figure 1. The search mechanism of PSO.

\subsection{Fitness Function}

Several techniques for handling constraints have been proposed in the specialized literature [25]. One of them considers the objective function penalization. Using this technique, the fitness function is formed by the objective function (1) plus penalty terms for particles that have violated some inequality constraints and equation constraints. Such fitness function can be expressed as:

$$
F_{i}=J_{i}+R_{1} \sum_{j=1}^{J}\left[g_{j}\right]^{2}+R_{2} \sum_{p=1}^{P}\left[h_{P}\right]^{2}
$$

where $R_{1}, R_{2}$ are coefficients of punishment, $g_{j}$ is value of violate inequality constraint, $h_{p}$ is value of violate equation constraint.

\subsection{Fuzzy Adaptive PSO}

In this section, a fuzzy adaptive particle swarm optimization will be described to fit a wider range of optimal problems. To design a fuzzy system to dynamically adapt the inertia weight, two variables are selected as inputs to the fuzzy system: the evolution speed factor and the square deviation of fitness for swarm; the output variable of system is the change of the inertia weight.

1) Evolution speed factor

The evolution speed factor $(E S F)$ measures the performance of the particle evolution process so far by the PSO. In case of a minimization problem, $G_{\text {best }}^{k} \geq G_{\text {best }}^{k+1}$, ESF is used as an input to bound the limit between 0 and 1 as:

$$
E S F=\frac{G_{b e s t}^{k+1}}{G_{\text {best }}^{k}}
$$

2) The square deviation of fitness

The square deviation of fitness the distribution of particles, it is by equation:

$$
\sigma=\sqrt{\frac{1}{n} \sum_{m=1}^{n}\left[\frac{F_{m}^{k}-F_{\text {mean }}^{k}}{\max \left\{F_{g}^{k}-F_{\text {mean }}^{k}, F_{\text {mean }}^{k}-F_{w}^{k}\right\}}\right]^{2}}
$$

where $F_{m}^{k}$ is fitness of individual $\mathrm{m}$ at iteration $k$, $F_{\text {mean }}^{k}$ is mean fitness of individuals at iteration $k, F_{g}^{k}$ is the best fitness of individual at iteration $k, F_{w}^{k}$ is the worst fitness of individual at iteration $k$.

3) Current inertia weight correction

The change of the inertia weight need both positive and negative corrections, this paper presents the range $(-0.04,+0.04)$ for the inertia weight correction $(\Delta \omega)$.

Two input variables and one output variable are defined to have three fuzzy sets: LOW (L), MIDDLE (M) and HIGH $(\mathrm{H})$ with associated membership function as left-Triangle, Triangle and right-Triangle, respectively in Figure 2.

The three membership functions are:

Left-triangle membership function: 


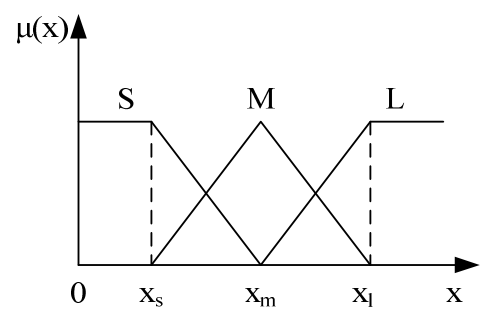

Figure 2. Triangular membership function.

$$
\mu_{1}=\left\{\begin{array}{cc}
1 & x<x_{s} \\
\frac{x_{m}-x}{x_{m}-x_{s}} & x_{s} \leq x \leq x_{m} \\
0 & x>x_{m}
\end{array}\right.
$$

Triangle membership function:

$$
\mu_{2}=\left\{\begin{array}{cc}
0 & x<x_{s} \\
\frac{x-x_{s}}{x_{m}-x_{s}} & x_{s} \leq x \leq x_{m} \\
\frac{x_{l}-x}{x_{l}-x_{m}} & x_{m}<x \leq x_{l} \\
0 & x>x_{l}
\end{array}\right.
$$

Right-triangle membership function:

$$
\mu_{3}=\left\{\begin{array}{cc}
0 & x<x_{m} \\
\frac{x-x_{m}}{x_{l}-x_{m}} & x_{m} \leq x \leq x_{l} \\
1 & x>x_{l}
\end{array}\right.
$$

The whole fuzzy system for dynamically adapting the inertia weight can be described as Table 1, where there are $9 \mathrm{IF} / \mathrm{THEN}$ rules for 2 input variables and 3 linguistic values of each input values. Degrees of membership of ESF $\left(\mu_{E S F}\right)$ and $\sigma\left(\mu_{\sigma}\right)$ are calculated from (18)-(20), respectively. Lasen product is used as fuzzy implication operator for the individual rules, using arithmetic product, the degree of fulfillment for rule $r$ is $D O F_{r}=\mu_{E S F}, \mu_{\sigma}$. For each rule, fuzzy inertia weight correction will be calculated by $D O F$. Finally, the center-of-area method is the most well known and simple defuzzification method which is implemented to determine the output crispy value [26]. The function of modify inertia weigh described as:

$$
\omega^{k+1}=\omega^{k}+\Delta \omega
$$

The proposed algorithm flowchart is depicted in Figure 3. The implementation steps are as follows:

Step 1. Initialization of the swarm

For a population size $n$, the particles are randomly

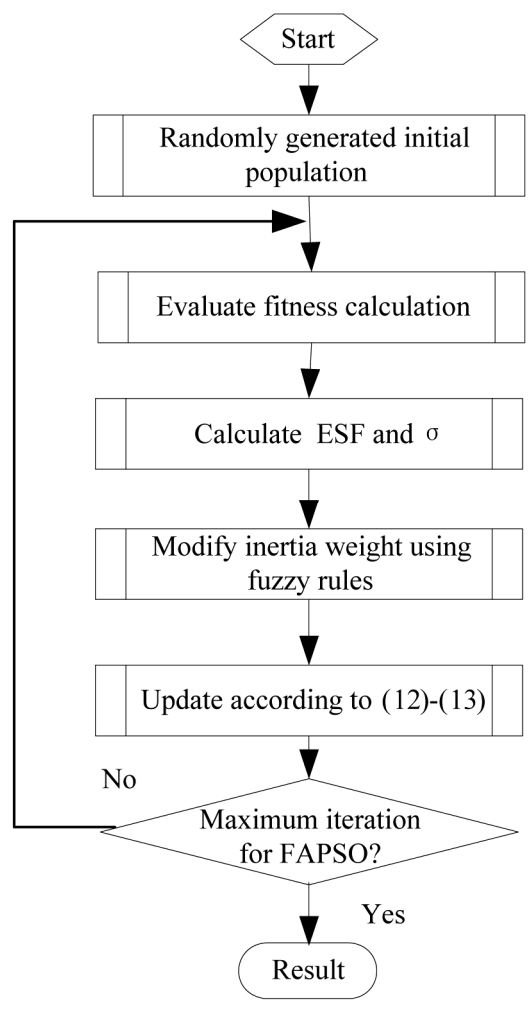

Figure 3. The framework flow chat of FAPSO.

Table 1. Fuzzy rules for inertia weight correction.

\begin{tabular}{cccc}
\hline \multirow{2}{*}{ Rule No. } & \multicolumn{2}{c}{ Input } & Output \\
\cline { 2 - 4 } & ESF & $\sigma$ & $\Delta \omega$ \\
\hline 1 & $\mathrm{~S}$ & $\mathrm{~S}$ & $\mathrm{~S}$ \\
2 & $\mathrm{~S}$ & $\mathrm{M}$ & $\mathrm{M}$ \\
3 & $\mathrm{~S}$ & $\mathrm{~L}$ & $\mathrm{~L}$ \\
4 & $\mathrm{M}$ & $\mathrm{S}$ & $\mathrm{L}$ \\
5 & $\mathrm{M}$ & $\mathrm{M}$ & $\mathrm{M}$ \\
6 & $\mathrm{M}$ & $\mathrm{L}$ & $\mathrm{S}$ \\
7 & $\mathrm{~L}$ & $\mathrm{~S}$ & $\mathrm{~S}$ \\
8 & $\mathrm{~L}$ & $\mathrm{M}$ & $\mathrm{S}$ \\
9 & $\mathrm{~L}$ & $\mathrm{~L}$ & $\mathrm{M}$ \\
\hline
\end{tabular}

generated in the range $0-1$ and located between the maximum and the minimum extreme.

1) Hydro plant

If there are $M$ reservoirs, the particles is initialized randomly within the effective real storage of reservoir, the $m$ th particle is represented as a matrix as follows:

$$
V_{h m}(t)=\left(v_{h}(1, t), v_{h}(2, t), \ldots, v_{h}(M, t)\right)
$$

The $j$ th reservoir is allocated a value of $V_{h j}$ as given 
below to satisfy the constraint given by (6).

$$
v_{h}(j, t)=V_{j, \min }+\operatorname{rand}() \times\left(V_{j, \max }-V_{j, \min }\right)
$$

The hydroelectric power outputs can easily be computed using (11).

2) Thermal plant

If there are $N$ thermal plants, the particles is initialized randomly within the effective real power generation limits, the $i$ th particle is represented as a matrix as follows:

$$
P_{t h i}(t)=\left(P_{t h}(1, t), P_{t h}(2, t), \ldots, P_{t h}(N, t)\right)
$$

\section{Step 2. Defining the fitness function}

FAPSO algorithm conventionally searches for the optimal solution by minimum a given fitness function. The hydrothermal co-ordination problem, the evaluation function is combinations of the thermal cost function and penalty function terms that take into account the various systems, unit and hydraulic network constraint violations. The evaluation function should be different in the feasible and infeasible search domains. The evaluation function is given by (15).

Step 3. Initialization of pbest and gbest

The fitness values obtained above for the initial particles, the best position of a particle is taken as pbest, and the best position of all pbests is taken as gbest.

\section{Step 4. Calculate ESF and $\sigma$}

When the best, the worst and mean of fitness values obtained, the evolution speed factor and square deviation of fitness can be used respectively Equations (16) and (17) calculated.

Step 5. Modify inertia weight using fuzzy rules

The inertia weight correction $(\Delta \omega)$ can be obtained using fuzzy rules, modify the inertia weight according to Equation (21).

\section{Step6. Update the swarm}

Modify the velocity vector of each particle using Equation (12), the particle position vector updated using (13).

\section{Step 7. Stopping criteria}

There are different criteria available to stop optimization algorithm. In this paper, maximum number of iterations is adopted as the stopping criterion.

\section{Simulation Result}

\subsection{Testing Strategies}

In this study, the maximum number of generations is set as 100 , the population size is set as 30 , and the general parameters of PSO are set as: $c_{1}=c_{2}=2$ for all the PSO runs, the same $\omega_{\max }=0.9, \omega_{\min }=0.3$ are employed for both FAPSO and PSO in order to examine their per- formance.

The program is implemented in MATLAB 7.0.1 and the simulations are carried on a Pentium IV personal computer with $256 \mathrm{M}$ RAM.

\subsection{Optimal Scheduling of Hydrothermal Power System}

The test hydrothermal power system consists of a multichain cascade of 4 hydro units and a number of thermal units represented by an equivalent thermal plant. The scheduling period is 24 hours, with an hour intervals.

The data of load demanded are shown as Table 2 . The system and unit parameters including costing functions and operating constraints are presented in [27].

FAPSO algorithm and PSO algorithm are used to simulate the operation course 20 times. The inflow course, water level-capacity curve, and the downstream stage discharge curve are all foregone. Table $\mathbf{3}$ gives the comparison results of FAPSO PSO and GA about two indexes: cost output and average execution time.

From Table 3, it is obvious that the results of FAPSO are best than that of PSO and GA with the object of the cost output and its convergence speed is very faster than PSO. The corresponding hourly hydro plant discharge is shown in Figure 4.

The results show that, among the three algorithms, the FAPSO offers the best solution quality, the proposed approach is superiority among its competitors.

Table 2. Data of load demanded (MW).

\begin{tabular}{cccccc}
\hline hour & load & hour & load & hour & load \\
\hline 1 & 1370 & 9 & 2240 & 17 & 2130 \\
2 & 1390 & 10 & 2320 & 18 & 2140 \\
3 & 1360 & 11 & 2230 & 19 & 2240 \\
4 & 1290 & 12 & 2310 & 20 & 2280 \\
5 & 1290 & 13 & 2230 & 21 & 2240 \\
6 & 1410 & 14 & 2200 & 22 & 2120 \\
7 & 1650 & 15 & 2130 & 23 & 1850 \\
8 & 2000 & 16 & 2070 & 24 & 1590 \\
\hline
\end{tabular}

Table 3. Comparison of results.

\begin{tabular}{ccc}
\hline Algorithm & Cost (\$) & Average time used (S) \\
\hline PSO & 921920 & 10.67 \\
FAPSO & 914660 & 4.73 \\
GA [27] & 926707 & - \\
\hline
\end{tabular}




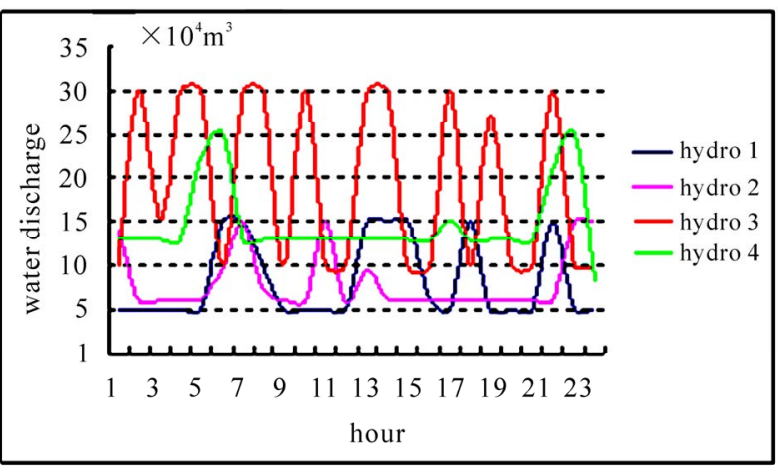

Figure 4. Hourly hydro plant discharge trajectories.

\section{Conclusions}

This paper presents an application of FAPSO for solving the hydrothermal optimal scheduling problem. FAPSO generates better solutions than other methods, mainly because it is implemented to dynamically adjust the inertia weight by using "IF-THEN" rules, this can improve the global and local search ability of the PSO and overcome the disadvantages of the PSO.

The proposed FAPSO is applied to the optimal operation of hydrothermal power plant. For comparison, simulations are conducted for FAPSO PSO and GA. The experimental results indicate that the proposed algorithm can improve the computational efficiency and produce more satisfactory output, which offers a new way to solve hydrothermal optimal operation problem.

\section{References}

[1] R. Ringlee, "Sensitivity Methods for Economic Dispatch of Hydroelectric Plants," IEEE Transactions on Automatic Control, Vol. 10, No. 3, 1965, pp. 315-322.

[2] T. N. Saha and S. A. Khapade, "An Application of a Direct Method for the Optimal Scheduling of Hydrothermal Power Systems," IEEE Transactions on Power Apparatus and Systems, Vol. 97, No. 3, 1978, pp. 977-985.

[3] H. Habibollahzadeh and J. A. Bubenko, "Application of Decomposition Techniques to Short Term Operation Planning of Hydro-Thermal Power System," IEEE Tran- sactions on PWRS, Vol. 1, No. 1, February 1986, pp. 41-47.

[4] M. Heidari, V. T. Chow, P. V. Kokotovic and D. D. Meredith, "Discrete Differential Dynamic Programming Approach to Water Resources Systems Optimization," Water Resources Research, Vol. 7, No. 2, 1971, pp. 273282.

[5] H. Laabs and R. Harboe, "Generation of Operating Rules with Stochastic Dynamic Programming and Multiple Objectives," Water Resources Management, Vol. 2, 1988, pp. 221-227.

[6] S.-C. Chang, C.-H. Chen, I.-K. Fong and P. B. Luh, "Hy- droelectric Generation Scheduling with an Effective Differential Dynamic Programming Algorithm," IEEE Transactions on Power System, Vol. 5, No. 3, 1990, pp. 737-743.

[7] D. P. Bertsekas, G. S. Lauer, N. R. Sandell and T. A. Posbergh, "Optimal Short-Term Scheduling of LargeScale Power Systems," IEEE Transactions on Automatic Contol, Vol. AC-28, No. 1, 1983, pp. 1-11.

[8] X. H. Guan, P. B. Luh and L. Zhang, "Nonlinear Approximation Method in Lagrangian Relaxation-Based Algorithms for Hydrothermal Scheduling," IEEE Transactions on Power System, Vol. 10, No. 2, May 1995, pp. 772-778.

[9] J. M. Ngundam, F. Kenfack and T. T. Tatietse, "Optimal Scheduling of Large-Scale Hydrothermal Power Systems Using the Lagrangian Relaxation Technique," International Journal of Electrical Power and Energy Systems, Vol. 22, No. 4, 2000, pp. 237-245.

[10] X. M. Bai and S. M. Shahidehpour, "Hydro-Thermal Scheduling by Tabu Search and Decomposition Method," IEEE Transactions on Power Systems, Vol. 11, No. 2, 1996, pp. 968-974.

[11] S. Li, S. M. Shahidehpour and C. Wang, "Promoting the Application of Expect Systems in Short-Term Unit Commitment," IEEE Transactions on Power System, Vol. 3, No. 1, May 1993, pp. 286-292.

[12] H. Sasaki, M. Watanabe, J. Kubokawa, N. Yorina and R. Yokoyama, "A Solution Method of Unit Commitment by Artificial Neural Network," Proceedings of 1991 IEEE Power Engineering Society Summer Meeting, Seattle, 1991.

[13] S. O. Orero and M. R. Irving,"Large Scale Unit Commitment Using a Hybrid Genetic Algorithms," International Journal of Electrical Power \& Energy Systems, Vol. 19, No. 1, 1997, pp. 45-55.

[14] W. P. Chang and X. J. Luo, "A Solution to the Unit Commitment Using Hybrid Genetic Algorithm," TENCON2008, IEEE Region 10 Conference, 19-21 November 2008

[15] C.-T. Cheng, W.-C. Wang, D.-M. Xu and K. W. Chau, "Optimizing Hydropower Reservoir Operation Using Hybrid Genetic Algorithm and Chaos," Water Resources Management, Vol. 22, No. 7, 2008, pp. 895-909.

[16] J. Kennedy and R. Eberhart, "Particle Swarm Optimization," Proceedings of IEEE International Conference Neural Networks, Perth, Vol. 4, 1995, pp. 1942-1948.

[17] M. A. Abido, "Optimal Design of Power-System Stabilizers Using Particle Swarm Optimization," IEEE Transactions on Energy Conversion, Vol. 17, No. 3, pp. 406-413.

[18] Z. L.Gaing, "A Particle Swarm Optimization Approach for Optimum Design of PID Controller in AVR System," IEEE Transactions on Energy Conversion, Vol. 19, No. 2, June 2004, pp. 384-391.

[19] B. Zhao, C. X. Guo and Y. J. Cao, "A Multiagent-Based Particle Swarm Optimization Approach for Optimal Reactive Power Dispatch," IEEE Transactions on Power 
System, Vol. 20, No. 2, May 2005, pp. 1070-1078.

[20] I. Selvakumar and K. Thanushkodi, "A New Particle Swarm Optimization Solution to Nonconvex Economic Dispatch Problems," IEEE Transactions on Power System, Vol. 22, No. 1, February 2007, pp. 42-51.

[21] K. T. Chaturvedi, M. Pandit and L. Srivastava, "SelfOrgianizing Hierachical Particle Swarm Optimization for Nonconvex Econmic Dispatch," IEEE Transactions on Power System, Vol. 23, No. 3, August 2008, pp. 10791087.

[22] Y. Shi and R. Eberhart, "A Modified Particle Swarm Optimize," Evolutionary Computation Proceedings, IEEE Congress on Computational Intelligence, Brisbane, 1998, pp. 69-73.

[23] Y. Shi and R. Eberhart, "Empirical Study of Particle Swarm Optimization," Proceedings of the IEEE Congress on Evolutionary Computation, 1999, pp. 1949-1950.
[24] J. Kenney and R. Eberhart, "Article Swarm Optimization," Proceedings of IEEE International Conference on Neural Networks, Perth, Vol. 5, 1995, pp. 2753-2756. http://www.engr.iupui.edu/shi/Conference/psopap4.html

[25] Z. Michalewicz and N. Attia, A. V. Sebald and L. J. Fogel, Eds., "Evolutionary Optimization of Constrained Problems," Proceedings of 3 rd Annual Conference on Evolutionary Programming, World Scientific, River Edge, 1994, pp. 98-108.

[26] E. H. Mamdani, "Application of Fuzzy Logic to Appromaximate Reasoning Using Linguistic Synthesis," IEEE Transactions on Computers, Vol. c-26, December 1977, pp. 1182-1191.

[27] S. O. Orero and M. R. Irving, "A Genetic Algorithm Modelling Framework and Solution Technique for Short Term Optimal Hydrothermal Scheduling," IEEE Transactions on Power System, Vol. 13, No. 2, May 1998. 